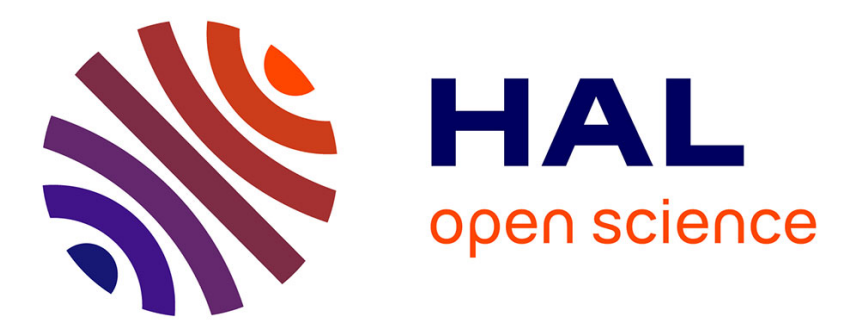

\title{
New imidazo[1,2-a]quinoxaline derivatives: Synthesis and in vitro activity against human melanoma
}

Carine Deleuze-Masquéfa, Georges Moarbess, Sonia Khier, Nadège David, Stéphanie Gayraud-Paniagua, Françoise Bressolle, Frédéric Pinguet, Pierre-Antoine Bonnet

\section{To cite this version:}

Carine Deleuze-Masquéfa, Georges Moarbess, Sonia Khier, Nadège David, Stéphanie GayraudPaniagua, et al.. New imidazo[1,2-a]quinoxaline derivatives: Synthesis and in vitro activity against human melanoma. European Journal of Medicinal Chemistry, 2009, 44 (9), pp.3406-3411. 10.1016/j.ejmech.2009.02.007 . hal-02309640

\section{HAL Id: hal-02309640 \\ https://hal.science/hal-02309640}

Submitted on 9 Oct 2019

HAL is a multi-disciplinary open access archive for the deposit and dissemination of scientific research documents, whether they are published or not. The documents may come from teaching and research institutions in France or abroad, or from public or private research centers.
L'archive ouverte pluridisciplinaire HAL, est destinée au dépôt et à la diffusion de documents scientifiques de niveau recherche, publiés ou non, émanant des établissements d'enseignement et de recherche français ou étrangers, des laboratoires publics ou privés. 
Provided for non-commercial research and education use. Not for reproduction, distribution or commercial use.

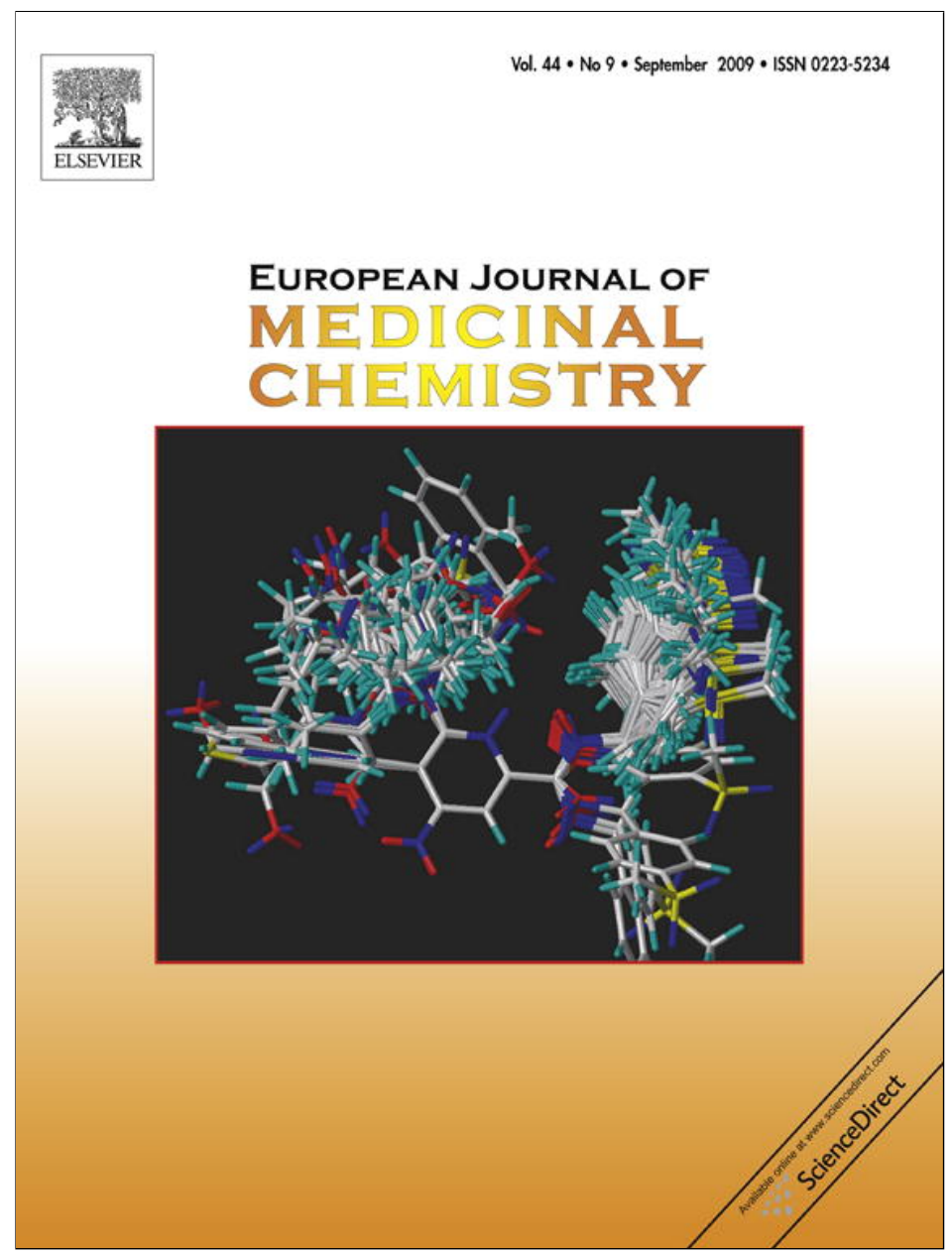

This article appeared in a journal published by Elsevier. The attached copy is furnished to the author for internal non-commercial research and education use, including for instruction at the authors institution and sharing with colleagues.

Other uses, including reproduction and distribution, or selling or licensing copies, or posting to personal, institutional or third party websites are prohibited.

In most cases authors are permitted to post their version of the article (e.g. in Word or Tex form) to their personal website or institutional repository. Authors requiring further information regarding Elsevier's archiving and manuscript policies are encouraged to visit:

http://www.elsevier.com/copyright 
Original article

\section{New imidazo[1,2-a]quinoxaline derivatives: Synthesis and in vitro activity against human melanoma}

\section{Carine Deleuze-Masquefa ${ }^{\mathrm{a}, *, 1}$, Georges Moarbess ${ }^{\mathrm{a}, 1}$, Sonia Khier $^{\mathrm{b}}$, Nadège David ${ }^{\mathrm{a}}$, Stéphanie Gayraud- Paniagua $^{a}$, Françoise Bressolle ${ }^{b, c}$, Frédéric Pinguet ${ }^{c}$, Pierre-Antoine Bonnet ${ }^{a}$}

${ }^{a}$ EA 4215, Laboratoire de Chimie des Biomolécules et de l'Environnement, Pharmacochimie et Biomolécules, Université Montpellier I, Faculté de Pharmacie, 15 av. Charles Flahault, BP 14491, 34093 Montpellier Cedex 5, France

${ }^{\mathrm{b}}$ Pharmacocinétique Clinique, Faculté de Pharmacie, Université Montpellier I, 15 av. Charles Flahault, BP 14491, 34093 Montpellier Cedex 5, France

${ }^{c}$ Centre Régional de Lutte contre le Cancer, Val d'Aurelle, Rue de la Croix Verte, 34000 Montpellier, France

\section{A R T I C L E I N F O}

\section{Article history:}

Received 14 October 2008

Received in revised form

26 January 2009

Accepted 12 February 2009

Available online 21 February 2009

\section{Keywords:}

Imidazoquinoxalines

Imiquimod

Melanoma

In vitro activity

\begin{abstract}
A B S T R A C T
New imidazo[1,2-a]quinoxaline analogues have been synthesized in good yields via a bimolecular condensation of 2-imidazole carboxylic acid, followed by a coupling with ortho-fluoroaniline and subsequent substitution on the imidazole ring by Suzuki Cross-coupling reaction using microwave assistance. Antitumor activities of these derivatives were evaluated by growth inhibition of A375 cells in vitro. All compounds exhibited high activities compared to imiquimod and fotemustine used as references.
\end{abstract}

() 2009 Elsevier Masson SAS. All rights reserved.

\section{Introduction}

In our previous studies [1-3], a general synthetic strategy has been elaborated which can easily get access to three basic heterocycles imidazo/pyrazoloquinoxalines. This method led us to the intermediates which remain non-substituted on the 5 -membered cycle with good yields. New imiquimod analogues in the imidazo[1,2-a]quinoxaline, imidazo[1,5-a]quinoxaline and pyrazolo[1,5-a]quinoxaline series have also been synthesised and tested in vitro and in vivo for their anti-cancer activities.

Imiquimod, an imidazoquinoline amine, has been initially approved for the topical treatment of external genital warts caused by human papilloma virus [4-6]. It has shown activities toward basal cell carcinomas (BCC), actinic keratoses, and some melanoma metastases [7-10]. Fotemustine is a third generation nitrosourea which is used both in melanoma and brain tumors [11]. However, its clinical application is somewhat limited both by its toxicity and by the resistance of melanoma cells [12]. BMS-345541, an imidazo[1,2a]quinoxaline derivative has shown important cytotoxic activities

\footnotetext{
* Corresponding author. Tel.: +33467548665/8640; fax: +33467548036. E-mail address: carine.masquefa@univ-montp1.fr (C. Deleuze-Masquefa).

1 Carine Deleuze-Masquefa and Georges Moarbess contributed equally to this work.
}

on melanoma in vitro and in vivo [13]. It was identified as a selective inhibitor of I $\kappa B$ kinase that blocked NF- $\kappa B$ pathway [14].

EAPB0203, a new imidazoquinoxaline amine derivative demonstrated significant in vitro activities against the human melanoma cell line A375, 110 and 45 times more active than fotemustine and imiquimod, respectively [1,2]. Tested in vivo in female Swiss Nude mice, EAPB0203 caused a significant decrease in tumor (M4Be xenografts) growth compared to vehicle control and fotemustine treatments [1,2].

Recently, EAPB0203 was evaluated in T-cell lymphomas and HTLV-I associated adult T-cell leukaemia/lymphoma. EAPB0203 exhibits an important cytotoxicity in vitro on HTLV-I-infected CD4 ${ }^{+}$ T-cell lines HuT-102, MT-2, and C91-PL as well as HTLV-1-negative malignant $\mathrm{CD}^{+}{ }^{+} \mathrm{T}$-cell lines CEM, Jurkat, HuT-78, and MOLT-4. The imidazoquinoxaline derivative exhibits inhibition of cell proliferation, G2/M cell cycle arrest, and induction of apoptosis in HTLV-I transformed and HTLV-I negative malignant T cells [15].

In continuation of our studies on the reactivity of nitrogen heterocycles, we focused on the preparation of imidazo[1,2-a]quinoxaline derivatives using the new synthetic strategy described previously $[1,2]$. To develop a convenient pathway in good agreement with rapid pharmacomodulation, we were interested in using the Suzuki Cross-coupling reaction. The application of this coupling to these series was reported on the bromo derivatives [16]. In this 
work, we turned our interest to study the incorporation of different arylboronic acids on the cytotoxic activity, in order to afford a variety of new derivatives of our lead compound EAPB0203.

\section{Results}

\subsection{Chemistry}

\subsubsection{Library synthesis}

For the preparation of the $1 H$-imidazo[1,2-a]quinoxaline derivatives, the procedure presented in Scheme 1 was used. Carbonylimidazole dimer 1 resulted from the bimolecular condensation of the 2-imidazole carboxylic acid, in presence of thionyl chloride in $95 \%$ overall yield. Product 1 was coupled with ortho-fluoroaniline to give the intermediate 2 , with a yield of $40 \%$. The tricyclic compound $\mathbf{3}$ was obtained via an intramolecular cyclization of $\mathbf{2}$ accomplished under strong basic conditions with $80 \%$ yield. Treatment of compound 3 with phosphorus oxychloride and $\mathrm{N}, \mathrm{N}$-diethylaniline yielded to $80 \%$ of compound $\mathbf{4}$ which was coupled with methylamine to give compound $\mathbf{5}$ with average yields of 93\% [1,3]. The bromination of compound $\mathbf{5}$ by $\mathrm{N}$-bromosuccinimide led to compound $\mathbf{6}$. The position of the bromine on the imidazole ring was confirmed by n.O.e study. Utilizing the appropriate arylbronic acid allowed for introduction of various groups on the imidazole ring of compound $\mathbf{6}$ via Suzuki reaction in the presence of palladium catalyst, basic conditions and under microwave assistance to give compounds $\mathbf{7 a - 7 n}$ in good yields.

\subsubsection{In vitro cytotoxic activity}

All compounds were evaluated for their cytotoxic activity in vitro against $A 375$ human melanoma cell line by comparison with imiquimod, fotemustine used as references and EAPB0203. Table 1 gives the $\mathrm{IC}_{50}$ values for all of the imidazo[1,2-a]quinoxaline derivatives as determined by the microculture tetrazolium dye assay. All the tested imidazo[1,2-a]quinoxalines caused a significant inhibition of cell growth, their cytotoxicities were superior to that of imiquimod and fotemustine. Compounds $7 \mathbf{d}, 7 \mathbf{k}-\mathbf{7 n}$ were considerably 10-100 times less active than the present EAPB0203 known to be 110 and 45 times more active than fotemustine and imiquimod on human melanoma cell line (A375), respectively [1,2]. Compounds $\mathbf{7 h}-\mathbf{7 j}$ showed a similar activity to EAPB0203. Five compounds $\mathbf{7 b}, \mathbf{7 c}, \mathbf{7 e - 7 g}$ are 7-9 times more potent against A375 than EAPB0203 (Table 1).

\section{Discussion}

The procedure presented in Scheme 1 was used to prepare the imidazo[1,2-a]quinoxaline derivatives. This method not only reduced the number of purification steps but also led us to propose a new common and efficient route for the preparation of the unsubstituted imidazo[1,2-a]quinoxalines on the imidazole core starting from the unsubstituted carboxylic acid. This new strategy also permitted the introduction of $R$ substituent at the last steps of the procedure with different commercial Suzuki cross-coupling reagents using microwave assistance.

The exact chemical structure of these imidazo[1,2-a]quinoxaline derivatives and the assignments of the position of substitution on the heterocycle using ${ }^{1} \mathrm{H}$ and ${ }^{13} \mathrm{C}$ NMR data were described previously [17].

In order to confirm the bromine position on the imidazole ring, we carried out a n.O.e study. The irradiation of $\mathrm{H}-9$ at $9.10 \mathrm{ppm}$ showed a correlation with only one proton $\mathrm{H}-8$ at $7.5 \mathrm{ppm}$ and the signal intensity of n.O.e effect was increased up to $15 \%$. No correlation was observed between $\mathrm{H}-9$ and protons of the imidazole ring. The same results were obtained by irradiation of $\mathrm{H}-2$ at $7.65 \mathrm{ppm}$. These results led us to confirm that the bromine was in the position 1 of the imidazole ring.

We reported previously [1,2] that EAPB0203 bearing phenethyle as substituent at position 1 was a lead compound with an activity against A375 cell line 110 times higher than fotemustine $\left(\mathrm{IC}_{50}=173 \mu \mathrm{M}\right)$ and 45 times higher than imiquimod $\left(\mathrm{IC}_{50}=70 \mu \mathrm{M}\right)$. In this paper, replacement of phenethyle (EAPB0203) by phenyle (compound 7a) on the imidazole ring resulted in no change in cytotoxicity. The presence of the carbon chain (EAPB0203) between the phenyl and imidazole core appeared to be less interesting, the activity was not influenced by the flexibility of the substituent on $R$ position. Introduction of an electron-donating group (compounds 7b, 7c, 7e, 7f) enhanced activity. This increase could be explained by additional interaction type hydrogen bound with the biological target. The substitution of an electron-withdrawing group on the phenyl core (compounds $\mathbf{7 k - 7 m}$ ) displayed decreased potency. The methoxy group at position 2 on the phenyl ring (compound $\mathbf{7 d}$ ) has proved to be less favorable than the 3 and 4 positions

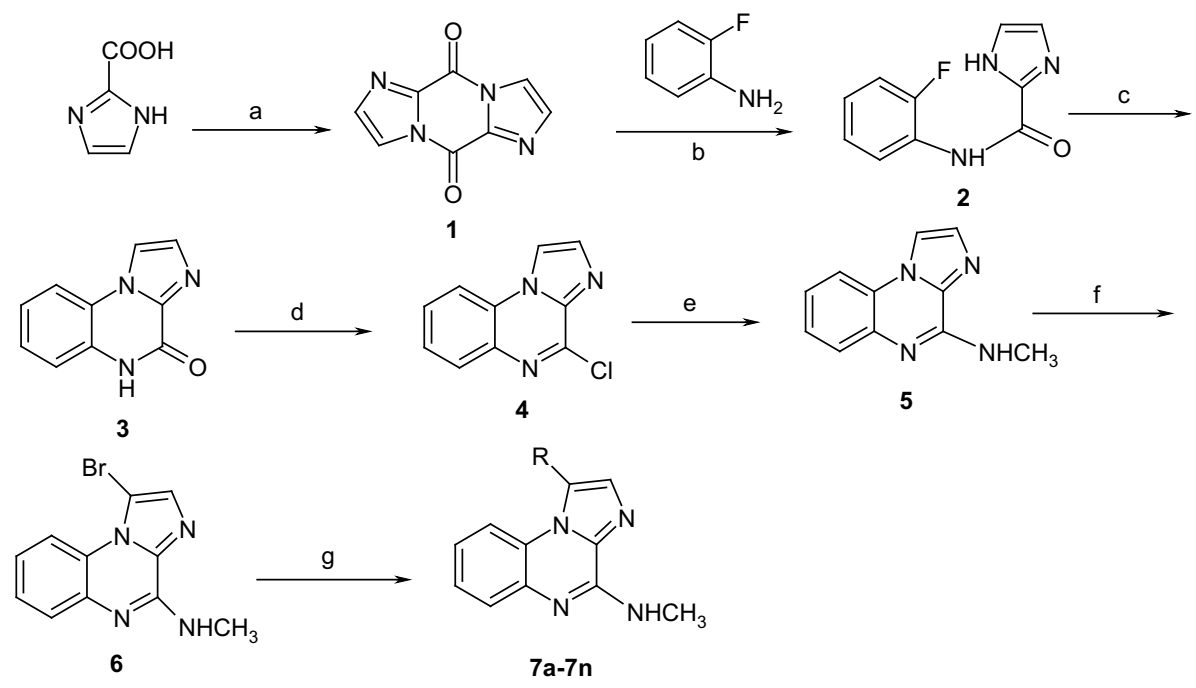

Scheme 1. Synthesis of $1 \mathrm{H}$-imidazo[1,2-a]quinoxaline derivatives. Reagents and conditions: (a) $\mathrm{SOCl}_{2}$ reflux, 18 h; (b) NaHMDS, THF, 5 h; (c) NaH, DMA, reflux, 10 h; (d) POCl,

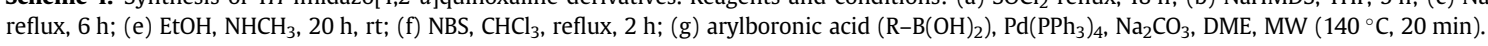


Table

Imidazo[1,2-a]quinoxaline derivatives: formula, $\mathrm{IC}_{50}$ against $\mathrm{A} 375$ (human melanoma cell lines), $\mathrm{p} K_{\mathrm{a}}$ and $\log P$ calculated values.

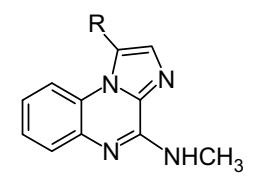

imidazo[1,2-a]quinoxaline

\begin{tabular}{|c|c|c|c|c|c|}
\hline Compounds & $\mathrm{R}_{1}$ & Formula & $\mathrm{IC}_{50}^{\mathrm{a}}(\mu \mathrm{M})$ & $\mathrm{p} K_{\mathrm{a}}^{*}$ & $\log P^{*}$ \\
\hline $7 a$ & $\mathrm{C}_{6} \mathrm{H}_{5^{-}}$ & $\mathrm{C}_{17} \mathrm{H}_{14} \mathrm{~N}_{4}$ & $2.19 \pm 0.08$ & $5.18 \pm 0.4$ & $3.68 \pm 1.43$ \\
\hline 7b (EAPB0503) & $3-\mathrm{OCH}_{3}-\mathrm{C}_{6} \mathrm{H}_{4}-$ & $\mathrm{C}_{18} \mathrm{H}_{16} \mathrm{~N}_{4} \mathrm{O}$ & $0.20 \pm 0.09$ & $5.10 \pm 0.4$ & $3.45 \pm 1.43$ \\
\hline $7 c$ & $4-\mathrm{OCH}_{3}-\mathrm{C}_{6} \mathrm{H}_{4}-$ & $\mathrm{C}_{18} \mathrm{H}_{16} \mathrm{~N}_{4} \mathrm{O}$ & $0.37 \pm 0.4$ & $5.45 \pm 0.4$ & $3.50 \pm 1.43$ \\
\hline 7d & $2-\mathrm{OCH}_{3}-\mathrm{C}_{6} \mathrm{H}_{4}-$ & $\mathrm{C}_{18} \mathrm{H}_{16} \mathrm{~N}_{4} \mathrm{O}$ & $122 \pm 25$ & $4.89 \pm 0.4$ & $3.35 \pm 1.43$ \\
\hline $7 e$ & $3-\mathrm{OC}_{2} \mathrm{H}_{5}-\mathrm{C}_{6} \mathrm{H}_{4}-$ & $\mathrm{C}_{19} \mathrm{H}_{18} \mathrm{~N}_{4} \mathrm{O}$ & $0.30 \pm 0.01$ & $5.11 \pm 0.4$ & $3.98 \pm 1.43$ \\
\hline $7 f$ & $3-\mathrm{OH}-\mathrm{C}_{6} \mathrm{H}_{4}-$ & $\mathrm{C}_{17} \mathrm{H}_{14} \mathrm{~N}_{4} \mathrm{O}$ & $0.56 \pm 0.12$ & $5.14 \pm 0.4$ & $2.94 \pm 1.43$ \\
\hline $7 g$ & $3-\mathrm{Br}-\mathrm{C}_{6} \mathrm{H}_{4}-$ & $\mathrm{C}_{17} \mathrm{H}_{13} \mathrm{BrN}_{4}$ & $0.65 \pm 0.02$ & $4.96 \pm 0.4$ & $4.43 \pm 1.43$ \\
\hline 7h & $3-\mathrm{CF}_{3}-\mathrm{C}_{6} \mathrm{H}_{4}-$ & $\mathrm{C}_{18} \mathrm{H}_{13} \mathrm{~F}_{3} \mathrm{~N}_{4}$ & $1.28 \pm 0.18$ & $4.86 \pm 0.4$ & $4.66 \pm 1.43$ \\
\hline $7 \mathbf{i}$ & $3-\mathrm{Cl}-\mathrm{C}_{6} \mathrm{H}_{4}-$ & $\mathrm{C}_{17} \mathrm{H}_{13} \mathrm{ClN}_{4}$ & $1.78 \pm 0.35$ & $4.88 \pm 0.4$ & $4.25 \pm 1.43$ \\
\hline $7 \mathbf{j}$ & $3-\mathrm{COOH}-\mathrm{C}_{6} \mathrm{H}_{4-}$ & $\mathrm{C}_{18} \mathrm{H}_{14} \mathrm{~N}_{4} \mathrm{O}_{2}$ & $3.47 \pm 0.55$ & $5.28 \pm 0.4$ & $3.36 \pm 1.43$ \\
\hline $7 k$ & $3-\mathrm{F}-\mathrm{C}_{6} \mathrm{H}_{4}-$ & $\mathrm{C}_{17} \mathrm{H}_{13} \mathrm{~F} \mathrm{~N}_{4}$ & $24.9 \pm 0.27$ & $4.88 \pm 0.4$ & $3.71 \pm 1.43$ \\
\hline 71 & $3-\mathrm{CN}-\mathrm{C}_{6} \mathrm{H}_{4}-$ & $\mathrm{C}_{18} \mathrm{H}_{13} \mathrm{~N}_{5}$ & $27.0 \pm 2.2$ & $4.83 \pm 0.4$ & $3.12 \pm 1.43$ \\
\hline $7 \mathrm{~m}$ & $3-\mathrm{NO}_{2}-\mathrm{C}_{6} \mathrm{H}_{4}-$ & $\mathrm{C}_{17} \mathrm{H}_{13} \mathrm{~N}_{5} \mathrm{O}_{2}$ & $40.0 \pm 3.9$ & $4.57 \pm 0.4$ & $3.21 \pm 1.43$ \\
\hline $7 n$ & $3-\left(\mathrm{C}_{4} \mathrm{H}_{3} \mathrm{O}\right)-$ & $\mathrm{C}_{15} \mathrm{H}_{12} \mathrm{~N}_{4} \mathrm{O}$ & $74.1 \pm 3.1$ & $5.21 \pm 0.4$ & $2.99 \pm 1.43$ \\
\hline ЕАРВ0203 & $\mathrm{C}_{6} \mathrm{H}_{5}-\left(\mathrm{CH}_{2}\right)_{2}-$ & $\mathrm{C}_{19} \mathrm{H}_{18} \mathrm{~N}_{4}$ & $1.57 \pm 0.56$ & $5.61 \pm 0.4$ & $4.91 \pm 1.42$ \\
\hline Imiquimod & & & $70.3 \pm 4.3$ & & \\
\hline Fotemustine & & & $173 \pm 24$ & & \\
\hline
\end{tabular}

${ }^{*} \mathrm{p} K_{\mathrm{a}}$ and $\log P$ values are calculated using the ACDlabs software.

${ }^{\mathrm{a}} \mathrm{IC}_{50}$, concentration of the compound $(\mu \mathrm{M})$ producing $50 \%$ cell growth inhibition after $96 \mathrm{~h}$ of drug exposure, as determined by the MTT assay. Each experiment was run at least three times, and the results are presented as average values \pm standard deviation.

(compounds 7b, 7c). No significant differences were observed between the $\mathrm{p} K_{\mathrm{a}}$ and $\log P$ calculated values (Table 1 ) of this series of compounds which are all substituted by an amino group.

In summary, a new series of imidazo[1,2-a]quinoxaline derivatives were synthesized with short reaction times by using microwave assistance and catalysts. These compounds were evaluated for their antitumor activities against human melanoma. These new analogues demonstrated interesting antitumor activities effect on A375 cell lines more potent than imiquimod and fotemustine. We found promising lead compounds (7b, 7c, 7e, 7f, 7g), EAPB0203 analogues, which exhibited high activities up to 9 times more potent for compound 7b (EAPB0503) than EAPB0203 (lead compound) on human melanoma cell lines. EAPB0503 can be considered as a new lead compound of the imidazo[1,2-a]quinoxaline series. Further studies are now in progress for the optimization of these compounds. On the other hand, in vivo studies will be complemented and documented to depict the antitumor activities of this new lead (EAPB0503) in female Swiss mice xenografted with human melanoma cell lines.

\section{Experimental}

\subsection{Chemistry}

All solvents and reagents were obtained from commercia sources and used without further purification unless indicated otherwise. ${ }^{1} \mathrm{H}$ and ${ }^{13} \mathrm{C}$ NMR spectra were recorded using a Bruker AC 300 spectrometer. Chemical shifts are reported in parts per million (ppm) from the tetramethylsilane resonance in the indicated solvent. Coupling constants are reported in $\operatorname{Hertz}(\mathrm{Hz})$, spectral splitting partners are designed as follow: singlet (s); doublet $(\mathrm{d})$; triplet $(\mathrm{t})$; multiplet $(\mathrm{m})$. Column chromatography was performed on Merck silica gel 60 (200-400 mesh). Elemental analysis was carried out at the Microanalytical Central Department (Montpellier, France). The microwave organic synthesis was assisted by the Biotage initiator (France). Melting points of solid compounds were measured by the Biocote apparatus. $\mathrm{p} K_{\mathrm{a}}$ and $\log P$ values are calculated using the ACDLabs software (Toronto, Canada).

\subsubsection{Diimidazo[1,2-a]piperazine-5,10-dione (1)}

The imidazole-2-carboxylic acid (5 g, $44.6 \mathrm{mmol}$ ) in suspension in thionyl chloride $(75 \mathrm{~mL})$ was heated to reflux, under agitation for $18 \mathrm{~h}$. The reaction mixture was cooled, then filtered, washed with toluene, and dried under high vacuum to obtain a yellow solid (yield, 90-95\%). M.p.: $164{ }^{\circ} \mathrm{C} .{ }^{1} \mathrm{H}$ NMR (300 MHz, DMSO- $\left.d_{6}\right) \delta: 8.85$ $(\mathrm{s}, 2 \mathrm{H}) ; 8.2(\mathrm{~s}, 2 \mathrm{H}) .{ }^{13} \mathrm{C}$ NMR (300 MHz, DMSO- $\left.d_{6}\right) \delta: 145.85,144.95$, 125.20, 120.15. Anal. calcd for $\mathrm{C}_{8} \mathrm{H}_{4} \mathrm{~N}_{4} \mathrm{O}_{2}$ : C, 51.07; $\mathrm{H}, 2.14 ; \mathrm{N}, 29.78$. Found: C, 51.15; H, 2.21; N, 29.46 .

\subsection{2. (2-Fluoroaniline)-1H-imidazole-4-carboxamide (2)}

To 2-fluoroaniline $(1.91 \mathrm{ml}, 19.77 \mathrm{mmol})$ in THF (13 ml), cooled in a $-10{ }^{\circ} \mathrm{C}$ bath, $(45.2 \mathrm{ml}, 45,2 \mathrm{mmol}, 1.0 \mathrm{M})$ sodium bis-(trimethylsilyl)amide in THF was added. The mixture was stirred for $1 \mathrm{~h}$, and a suspension of $\mathbf{1}(1.77 \mathrm{~g}, 9.4 \mathrm{mmol})$ in THF $(20 \mathrm{~mL})$ was added and allowed to warm to room temperature. The mixture was stirred for $2 \mathrm{~h}$, and acetic acid was added to $\mathrm{pH}$ 7. The reaction mixture was concentrated in vacuum followed by the addition of water and saturated $\mathrm{NaHCO}_{3}$. The solid was collected by filtration, washed with water and cyclohexane, and dried under high vacuum to give 2 as a beige solid (yield, $40 \%)$. M.p.: $250{ }^{\circ} \mathrm{C} .{ }^{1} \mathrm{H}$ NMR $(300 \mathrm{MHz}$, DMSO- $\left.d_{6}\right) \delta: 8.12(\mathrm{dd}, 1 \mathrm{H}), 7.92(\mathrm{dd}, 1 \mathrm{H}), 6.92(\mathrm{t}, 1 \mathrm{H}), 7.13(\mathrm{t}, 1 \mathrm{H}), 7.07$ $(\mathrm{s}, 2 \mathrm{H}) .{ }^{19} \mathrm{~F}$ NMR: $126 .{ }^{13} \mathrm{C}$ NMR $\left(300 \mathrm{MHz}\right.$, DMSO-d $\left.d_{6}\right) \delta: 151.69$, 150.92, 150.52, 130.14, 128.79, 126.96, 123.97, 121.03, 119.53, 115.03. Anal. calcd for $\mathrm{C}_{10} \mathrm{H}_{8} \mathrm{FN}_{3} \mathrm{O}: \mathrm{C}, 58.54 ; \mathrm{H}, 3.93 ; \mathrm{N}, 20.48 \mathrm{C}, 64,37 ; \mathrm{H}$, 6.13; N, 16.09. Found: C, 58.33; H, 3.55; N, 20.14 .

\subsubsection{Imidazo[1,2-a/quinoxaline-6-(5H)-one (3)}

A mixture of $2(2 \mathrm{~g}, 9.75 \mathrm{mmol})$ and sodium hydride $(0.3 \mathrm{~g}$, $1.17 \mathrm{mmol})$ in dimethylacetamide $(80 \mathrm{~mL})$ was heated to reflux for $6 \mathrm{~h}$. The reaction mixture was then concentrated in vacuum followed by the addition of water and saturated ammonium chloride. The solid was collected by filtration, washed with water, and dried to give 3 (yield, 80\%). M.p.: $322{ }^{\circ} \mathrm{C} .{ }^{1} \mathrm{H}$ NMR (300 MHz, DMSO- $d_{6}$ ) 
$\delta: 9.4(\mathrm{~s}, 1 \mathrm{H}), 8.2(\mathrm{~d}, 1 \mathrm{H}), 7.8(\mathrm{~d}, 1 \mathrm{H}), 7.31(\mathrm{~d}, 1 \mathrm{H}), 7.1-7.3(\mathrm{~m}, 3 \mathrm{H})$. ${ }^{13} \mathrm{C}$ NMR (300 MHz, DMSO-d $\left.)_{6}\right) \delta: 149.26,139.11,137.89,132.43$, $126.43,123.94,123.02,119.40,118.17,115.42$. Anal. calcd for $\mathrm{C}_{10} \mathrm{H}_{7} \mathrm{~N}_{3} \mathrm{O}: \mathrm{C}, 64.86 ; \mathrm{H}, 3.81 ; \mathrm{N}, 22.69$. Found: C, 64.44; $\mathrm{H}, 4.03 ; \mathrm{N}$, 22.98 .

\subsubsection{6-Chloroimidazo[1,2-a]quinoxaline (4)}

To $3(1.42 \mathrm{~g}, 7.7 \mathrm{mmol})$, phosphorus oxychloride $(10 \mathrm{~mL})$ and $\mathrm{N}, \mathrm{N}$-diethylaniline $(1.6 \mathrm{~mL})$ were added and the mixture was heated to reflux for $2 \mathrm{~h}$. The reaction mixture was concentrated under vacuum, and the residue was cooled in an ice bath. Water was added to the residue and neutralized with saturated $\mathrm{NaHCO}_{3}$. The solid was collected by filtration and purified by recrystallisation from methanol to yield 4 as a white solid (yield, 80\%). M.p.: $188^{\circ} \mathrm{C}$. ${ }^{1} \mathrm{H}$ NMR $\left(300 \mathrm{MHz}\right.$, DMSO- $\left.d_{6}\right) \delta: 8.5(\mathrm{~s}, 1 \mathrm{H}), 8.06(\mathrm{~d}, 1 \mathrm{H}), 7.56(\mathrm{~s}, 1 \mathrm{H})$, $7.35(\mathrm{~m}, 1 \mathrm{H}), 7.25(\mathrm{~m}, 2 \mathrm{H}) .{ }^{13} \mathrm{C}$ NMR $\left(300 \mathrm{MHz}\right.$, DMSO- $\left.d_{6}\right) \delta: 135.82$, $132.19,131.75,130.4,129.95,127.12,125.03,115.67,113.4$. Anal. calcd for $\mathrm{C}_{10} \mathrm{H}_{6} \mathrm{~N}_{3} \mathrm{Cl}$ : C, 58.98; $\mathrm{H}, 2.97 ; \mathrm{N}, 20.64$. Found: C, 59.12; H, 2.76; $\mathrm{N}, 20.45$.

\subsubsection{N-Methylimidazo[1,2-a]quinoxalin-4-amine (5)}

Methylamine of a $40 \%(\mathrm{w} / \mathrm{v})$ aqueous solution, was added dropwise to a stirred solution of $4(0.110 \mathrm{~g}, 0.54 \mathrm{mmol})$ in absolute EtOH $(10 \mathrm{~mL})$ at room temperature. After $40 \mathrm{~h}$, another portion of methylamine $(0.15 \mathrm{ml}, 1.74 \mathrm{mmol})$ of a $40 \%(\mathrm{w} / \mathrm{v})$ aqueous solution was added and stirring was maintained for additional $3 \mathrm{~h}$. The solvent was removed under reduced pressure, and the residue was dissolved in $\mathrm{CH}_{2} \mathrm{Cl}_{2}(10 \mathrm{~mL})$. The organic fraction was successively washed with $5 \% \mathrm{NaHCO}_{3}(15 \mathrm{~mL})$ and water $(15 \mathrm{~mL})$, dried $\left(\mathrm{Na}_{2} \mathrm{SO}_{4}\right)$, and concentrated under reduced pressure. The product was purified by column chromatography on silica gel with $\mathrm{C}_{6} \mathrm{H}_{12}-$ EtOAc (70:30, v/v) as eluent to yield a white solid (yield, 93\%). M.p.: $180{ }^{\circ} \mathrm{C} .{ }^{1} \mathrm{H}$ NMR $\left(300 \mathrm{MHz}\right.$, DMSO-d $\left.d_{6}\right) \delta: 7.92(\mathrm{~s}, 1 \mathrm{H}), 7.75(\mathrm{dd}, 1 \mathrm{H})$, 7,65 (dd, 1H), 7.52 (s, 1H), $7.4(\mathrm{t}, 1 \mathrm{H}), 7.25(\mathrm{t}, 1 \mathrm{H}), 6.15(\mathrm{~s}, 1 \mathrm{H}), 3.25$ (d, $3 \mathrm{H}) .{ }^{13} \mathrm{C}$ NMR (300 MHz, DMSO-d $\left.d_{6}\right) \delta: 142.25,139.17,131.99,129.4$, $128.17,127.2,125.14,124.34,114.14,113.89,29.20$. Anal. calcd for $\mathrm{C}_{11} \mathrm{H}_{10} \mathrm{~N}_{4} \mathrm{C}, 66.65 ; \mathrm{H}, 5.08$; N, 28.26. Found: C, 66.26; H, 5.53; N, 28.23 .

\subsubsection{1-Bromo-N-methylimidazo[1,2-a]quinoxalin-4-amine (6)}

A solution of $5(1.5 \mathrm{~g}, 7.5 \mathrm{mmol})$ and $N$-bromosuccinimide $(1.5 \mathrm{~g}$, $7.5 \mathrm{mmol})$ in $\mathrm{CHCl}_{3}(200 \mathrm{~mL})$ was heated under reflux for $2 \mathrm{~h}$. The resulting solution was cooled, washed with $5 \%$ sodium hydrogen carbonate $(50 \mathrm{~mL})$, dried and concentrated. The residue was purified by recristallisation from chloroform to a white solid $(1.18 \mathrm{~g}$, 67\%). M.p.: $202{ }^{\circ} \mathrm{C} .{ }^{1} \mathrm{H}$ NMR (300 MHz, DMSO-d 6 ) $\delta: 9.10$ (s, $\left.1 \mathrm{H}\right), 7.75$ $(\mathrm{dd}, 1 \mathrm{H}), 7,65(\mathrm{dd}, 1 \mathrm{H}), 7.4(\mathrm{t}, 1 \mathrm{H}), 7.25(\mathrm{t}, 1 \mathrm{H}), 6.15(\mathrm{~s}, 1 \mathrm{H}), 3.25(\mathrm{~d}$, $3 \mathrm{H}) .{ }^{13} \mathrm{C}$ NMR $(300 \mathrm{MHz}$, DMSO-d $) \delta: 141.97,140.54,138.85,128.96$, $127.78,127.09,126.03,125.13,113.86,101.4,29.20$. Anal. calcd for $\mathrm{C}_{11} \mathrm{H}_{9} \mathrm{~N}_{4} \mathrm{Br}$ : C, 47.68; H, 3.27; N, 20.22. Found: C, 47.33; H, 3.53; N, 20.53 .

\subsection{General procedure for the Suzuki reaction}

To a mixture of 6 (300 mg, $1.08 \mathrm{mmol})$ and $\mathrm{Pd}\left(\mathrm{PPh}_{3}\right)(63 \mathrm{mg}$, $0.05 \mathrm{mmol})$ in DME $(15 \mathrm{~mL})$ was added the corresponding aryl boronic acid followed by the addition of sodium carbonate $(234 \mathrm{mg})$ in water $(5 \mathrm{~mL})$. The reaction was irradiated in a sealed tube at $140^{\circ} \mathrm{C}$ for $20 \mathrm{~min}$ using a Biotage Initiator microwave synthesizer. The reaction mixture was poured on water and then extracted with dichloromethane $(2 \times 40 \mathrm{~mL})$. The combined organic extracts were washed with water $(40 \mathrm{~mL})$, dried and concentrated to dryness under vacuo. The crude product was purified by column chromatography (silica gel eluting with dichloromethane).
4.2.1. N-Methyl-1-phenylimidazo[1,2-a]quinoxalin-4-amine (7a)

Phenylboronic acid (260 mg, $2.13 \mathrm{mmol}$ ). White solid (90\%). M.p.: $186^{\circ}{ }^{\circ} .{ }^{1} \mathrm{H}$ NMR $\left(300 \mathrm{MHz}, \mathrm{CDCl}_{3}\right) \delta: 8.15(\mathrm{~s}, 1 \mathrm{H}), 7.99(\mathrm{dd}, 1 \mathrm{H})$, $7.52(\mathrm{~m}, 8 \mathrm{H}), 5.7(\mathrm{~s}, 1 \mathrm{H}), 3.01(\mathrm{~d}, 3 \mathrm{H}) .{ }^{13} \mathrm{C} \mathrm{NMR}\left(300 \mathrm{MHz}, \mathrm{CDCl}_{3}\right) \delta$ : $142.86,139.37,131.13,130.74,129.67,129.02,126.77,125.75,124.90$, $124.78,124.47,121.12,114.96,29.20$. Anal. calcd for $\mathrm{C}_{17} \mathrm{H}_{14} \mathrm{~N}_{4}$ : C, 74.43; H, 5.14; N, 20.42. Found: C, 74.15; H, 5.46; N, 20.09.

\subsubsection{1-(3-Methoxyphenyl)-N-methylimidazo[1,2-a]quinoxalin-4-} amine (7b) (EAPB0503)

3-Methoxyphenylboronic acid (329 mg, $2.16 \mathrm{mmol}$ ). White solid (90\%). M.p.: $168{ }^{\circ} \mathrm{C} .{ }^{1} \mathrm{H}$ NMR (300 MHz, $\left.\mathrm{CDCl}_{3}\right) \delta: 9.04$ (s, $\left.1 \mathrm{H}\right), 8$ (dd, $1 \mathrm{H}), 7.82(\mathrm{~m}, 2 \mathrm{H}), 7.4(\mathrm{~m}, 3 \mathrm{H}), 6.99(\mathrm{~d}, 1 \mathrm{H}), 6.86(\mathrm{~s}, 1 \mathrm{H}), 5.7(\mathrm{~s}, 1 \mathrm{H})$, $3.83(\mathrm{~s}, 3 \mathrm{H}), 2.88(\mathrm{~d}, 3 \mathrm{H}) .{ }^{13} \mathrm{C}$ NMR $\left(300 \mathrm{MHz} \mathrm{CDCl}_{3}\right) \delta: 160.98$, 142.86, 139.57, 131.13, 130.56, 129.5, 129.02, 128.4, 127.39, 124.9, 124.40, 122.10, 118.02, 114.96, 111.87, 108.08, 55.30, 29.20. Anal. calcd for $\mathrm{C}_{18} \mathrm{H}_{16} \mathrm{~N}_{4} \mathrm{O}$ : C, 71.04; $\mathrm{H}, 5.30 ; \mathrm{N}, 18.41$. Found: $\mathrm{C}, 71.40 ; \mathrm{H}$, 5.66; N, 18.09 .

4.2.3. 1-(4-Methoxyphenyl)-N-methylimidazo[1,2-a]quinoxalin-4amine (7c)

4-Methoxyphenylboronic acid (329 mg, $2.16 \mathrm{mmol}$ ). White solid (95\%). M.p.: $198{ }^{\circ} \mathrm{C} .{ }^{1} \mathrm{H}$ NMR $\left(300 \mathrm{MHz}, \mathrm{CDCl}_{3}\right) \delta: 8.14(\mathrm{~s}, 1 \mathrm{H}), 8.00$ $(\mathrm{d}, 1 \mathrm{H}), 7.67(\mathrm{~m}, 3 \mathrm{H}), 7.5(\mathrm{~m}, 2 \mathrm{H}), 7.05(\mathrm{~m}, 2 \mathrm{H}), 5.77(\mathrm{~s}, 1 \mathrm{H}), 3.82(\mathrm{~s}$, $3 \mathrm{H}), 2.99(\mathrm{~s}, 3 \mathrm{H}) .{ }^{13} \mathrm{C}$ NMR $\left(300 \mathrm{MHz}, \mathrm{CDCl}_{3}\right) \delta$ : 158.05, 142.86, 139.57, 131.13, 129.61, 129.02, 125.96, 125.68, 124.47, 124.02, 121.12, 114.96, 113.35, 57.83, 29.20. Anal. calcd for $\mathrm{C}_{18} \mathrm{H}_{16} \mathrm{~N}_{4} \mathrm{O}$ : C, 71.04; $\mathrm{H}$, 5.30; N, 18.41. Found: C, 71.18; H, 5.58; N, 18.13.

\subsubsection{1-(2-Methoxyphenyl)-N-methylimidazo[1,2-a]quinoxalin-4- amine (7d)}

2-Methoxyphenylboronic acid (329 mg, $2.16 \mathrm{mmol}$ ). Beige solid (94\%). M.p.: $180{ }^{\circ} \mathrm{C} .{ }^{1} \mathrm{H}$ NMR $\left(300 \mathrm{MHz}, \mathrm{CDCl}_{3}\right) \delta: 8.1(\mathrm{~d}, 1 \mathrm{H}), 7.60(\mathrm{~m}$, $3 \mathrm{H}), 7.48(\mathrm{t}, 1 \mathrm{H}), 7.30(\mathrm{~m}, 4 \mathrm{H}), 5.74(\mathrm{~s}, 1 \mathrm{H}), 3.85(\mathrm{~s}, 3 \mathrm{H}), 3.00(\mathrm{~s}, 3 \mathrm{H})$. ${ }^{13} \mathrm{C}$ NMR $\left(300 \mathrm{MHz}, \mathrm{CDCl}_{3}\right) \delta: 153.81,142.74,139.57,131.64,131.13$, 129.02, 128.44, 127.58, 124.45, 124.28, 121.37, 118.02, 114.86, 11.98, 55.32, 29.20. Anal. calcd for $\mathrm{C}_{18} \mathrm{H}_{16} \mathrm{~N}_{4} \mathrm{O}$ : C, 71.04; H, 5.30; N, 18.41. Found: C, 70.98; H, 5.12; N, 18.22 .

\subsubsection{1-(3-Ethoxyphenyl)- N-methylimidazo[1,2-a]quinoxalin-4- amine (7e)}

3-Ethoxyphenylboronic acid (360 mg, $2.17 \mathrm{mmol})$. White solid (52\%). M.p.: $162{ }^{\circ} \mathrm{C} .{ }^{1} \mathrm{H}$ NMR (300 MHz, $\left.\mathrm{CDCl}_{3}\right) \delta: 8.34(\mathrm{~s}, 1 \mathrm{H}), 8.02$ $(\mathrm{d}, 1 \mathrm{H}), 7.83(\mathrm{~d}, 1 \mathrm{H}), 7.67(\mathrm{t}, 1 \mathrm{H}), 7.5(\mathrm{~m}, 2 \mathrm{H}), 6.96(\mathrm{~d}, 1 \mathrm{H}), 6.85(\mathrm{~s}, 1 \mathrm{H})$, $5.74(\mathrm{~s}, 1 \mathrm{H}), 3.89(\mathrm{~m}, 2 \mathrm{H}), 2.89(\mathrm{~s}, 3 \mathrm{H}), 1.38(\mathrm{t}, 3 \mathrm{H}) .{ }^{13} \mathrm{C} \mathrm{NMR}$ $\left(300 \mathrm{MHz}, \mathrm{CDCl}_{3}\right) \delta: 161.36,142.86,139.57,131.13,130.22,129.5$, 129.02, 128.41, 127.05, 124.9, 122.1, 118.02, 114.96, 113.77, 108.12, 63.8, 29.2, 15.2. Anal. calcd for $\mathrm{C}_{19} \mathrm{H}_{18} \mathrm{~N}_{4} \mathrm{O}$ : C, 71.68; $\mathrm{H}, 5.70 ; \mathrm{N}, 17.60$. Found: C, 71.43; H, 5.56; N, 17.39 .

\subsubsection{1-(3-Hydroxyphenyl)-N-methylimidazo[1,2-a]quinoxalin-4- amine (7f)}

3-Hydroxyphenylboronic acid (329 mg, $2.16 \mathrm{mmol}$ ). White solid (85\%). M.p.: $278{ }^{\circ} \mathrm{C} .{ }^{1} \mathrm{H}$ NMR (300 MHz, $\left.\mathrm{CDCl}_{3}\right) \delta: 8.33$ (s, $\left.1 \mathrm{H}\right), 7.99$ $(\mathrm{d}, 1 \mathrm{H}), 7.79(\mathrm{~d}, 1 \mathrm{H}), 7.70(\mathrm{~m}, 2 \mathrm{H}), 7.67(\mathrm{t}, 1 \mathrm{H}), 7.50(\mathrm{~m}, 3 \mathrm{H}), 6.857(\mathrm{~m}$, $2 \mathrm{H}), 3.02(\mathrm{~s}, 3 \mathrm{H}) .{ }^{13} \mathrm{C}$ NMR $\left(300 \mathrm{MHz}, \mathrm{CDCl}_{3}\right) \delta$ : 160.07, 142.86, 139.57, 131.46, 131.13, 130.35, 129.63, 129.02, 127.64, 126.78, 125.39, $124.9,124.40,122.83,120.71,114.86,114.54,29.02$. Anal. calcd for $\mathrm{C}_{17} \mathrm{H}_{14} \mathrm{~N}_{4} \mathrm{O}$ : C, 70.33; H, 4.86; N, 19.30. Found: C, 70.49; H, 5.19; N, 19.09 .

\subsubsection{1-(3-Bromophenyl)- N-methylimidazo[1,2-a]quinoxalin-4- amine $(\mathbf{7 g})$}

3-Bromophenylboronic acid (435 mg, $2.16 \mathrm{mmol}$ ). Yellow solid (78\%). M.p.: $144{ }^{\circ} \mathrm{C} .{ }^{1} \mathrm{H}$ NMR (300 MHz, $\left.\mathrm{CDCl}_{3}\right) \delta: 8.18$ (s, 1H), 7.90 
(m, 2H), $7.67(\mathrm{t}, 1 \mathrm{H}), 7.50(\mathrm{~m}, 2 \mathrm{H}), 7.45(\mathrm{~m}, 3 \mathrm{H}), 5.74(\mathrm{~s}, 1 \mathrm{H}), 2.98(\mathrm{~s}$, 3H). ${ }^{13} \mathrm{C}$ NMR $\left(300 \mathrm{MHz}, \mathrm{CDCl}_{3}\right) \delta: 142.86,139.57,132.86,131.13$, $130.53,129.63,129.03,127.64,126.78,125.39,124.90,124.30$, 122.93, 120.71, 114.96, 29.20. Anal. calcd for $\mathrm{C}_{17} \mathrm{H}_{13} \mathrm{Br} \mathrm{N} \mathrm{N}_{4}$ : C, 57.81; H, 3.71; N, 15.86. Found: C, 58.05; H, 3.55; N, 15.99.

\subsubsection{1-(3-Trifluoromethylphenyl)- N-methylimidazo[1,2-} a)quinoxalin-4-amine ( $7 \boldsymbol{h})$

3-Trifluoromethylphenylboronic acid (411 mg, $2.16 \mathrm{mmol}$ ) White solid (98\%). M.p.: $210{ }^{\circ} \mathrm{C} .{ }^{1} \mathrm{H}$ NMR $\left(300 \mathrm{MHz}, \mathrm{CDCl}_{3}\right) \delta: 8.14(\mathrm{~s}$, $1 \mathrm{H}), 8.10(\mathrm{~s}, 1 \mathrm{H}), 8.02(\mathrm{~m}, 2 \mathrm{H}), 7.85(\mathrm{~d}, 1 \mathrm{H}), 7.60(\mathrm{~m}, 2 \mathrm{H}), 7.48(\mathrm{t}, 1 \mathrm{H})$, $5.74(\mathrm{~s}, 1 \mathrm{H}), 2.96(\mathrm{~s}, 3 \mathrm{H}) .{ }^{13} \mathrm{C}$ NMR $\left(300 \mathrm{MHz}, \mathrm{CDCl}_{3}\right) \delta: 142.86$, 139.57, 132.62, 132.30, 132.02, 131.60, 131.13, 129.02, 128.86, 124.90, $124.47,122.51,121.59,120.40,114.86,114.54,29.02$. Anal. calcd for $\mathrm{C}_{18} \mathrm{H}_{13} \mathrm{~N}_{4} \mathrm{~F}_{3}$ : C, 63.16; H, 3.83; N, 16.65. Found: C, 63.03; H, 3.76; N, 16.74 .

\subsubsection{1-(3-Chlorophenyl)-N-methylimidazo[1,2-a]quinoxalin-4- amine (7i)}

3-Chlorophenylboronic acid (339 mg, $2.16 \mathrm{mmol}$ ). Yellow solid (63\%). M.p.: $188^{\circ} \mathrm{C} .{ }^{1} \mathrm{H}$ NMR (300 MHz, $\left.\mathrm{CDCl}_{3}\right) \delta: 8.48$ (s, $\left.1 \mathrm{H}\right), 8.02$ $(\mathrm{d}, 1 \mathrm{H}), 7.67(\mathrm{t}, 1 \mathrm{H}), 7.58(\mathrm{~m}, 2 \mathrm{H}), 7.49(\mathrm{~m}, 3 \mathrm{H}), 7.10(\mathrm{~s}, 1 \mathrm{H}), 5.74(\mathrm{~s}$, $1 \mathrm{H}), 2.81(\mathrm{~s}, 3 \mathrm{H}) .{ }^{13} \mathrm{C}$ NMR $\left(300 \mathrm{MHz}, \mathrm{CDCl}_{3}\right) \delta: 142.86,139.57$, $135.86,131.13,130.50,130.16,129.76,129.36,129.02,126.68,124.9$, $124.46,123.39,122.96,121.74,114.96,29.15$. Anal. calcd for $\mathrm{C}_{17} \mathrm{H}_{13} \mathrm{ClN}_{4}$ : C, 66.13; H, 4.24; N, 11.48. Found: C, 66.35; H, 4.01; N, 11.25.

4.2.10. 1-(3-Carboxyphenyl)- N-methylimidazo[1,2-a]quinoxalin-4amine $(\mathbf{7 j})$

3-Carboxyphenylboronic acid (357 $\mathrm{mg}, 2.16 \mathrm{mmol}$ ). Orange solid (87\%). M.p.: $130{ }^{\circ} \mathrm{C} .{ }^{1} \mathrm{H}$ NMR $\left(300 \mathrm{MHz}, \mathrm{CDCl}_{3}\right) \delta: 8.41(\mathrm{~d}, 1 \mathrm{H})$, $8.26(\mathrm{~s}, 1 \mathrm{H}), 8.01(\mathrm{~m}, 2 \mathrm{H}), 7.80(\mathrm{~m}, 4 \mathrm{H}), 7.58(\mathrm{~m}, 2 \mathrm{H}), 7.47(\mathrm{t}, 1 \mathrm{H}), 2.95$ (s, 3H). ${ }^{13} \mathrm{C}$ NMR $\left(300 \mathrm{MHz}, \mathrm{CDCl}_{3}\right) \delta: 167.35,142.86,139.54,131.88$, 131.13, 130.34, 129.02, 128.76, 127.1, 126.72, 124.9, 124.47, 122.51, 122.38, 114.96, 29.20. Anal. calcd for $\mathrm{C}_{18} \mathrm{H}_{14} \mathrm{~N}_{4} \mathrm{O}_{2}$ : C, 67.92; $\mathrm{H}, 4.43$; N, 17.60. Found: C, 67.72; H, 4.35; N, 17.63 .

\subsubsection{1-(3-Fluorophenyl)- N-methylimidazo[1,2-a]quinoxalin-4-} amine $(\mathbf{7 k})$

3-Fluorophenylboronic acid (303 mg, $2.16 \mathrm{mmol}$ ). Yellow solid (66\%). M.p.: $220{ }^{\circ} \mathrm{C} .{ }^{1} \mathrm{H}$ NMR (300 MHz, $\left.\mathrm{CDCl}_{3}\right) \delta: 8.22$ (s, $\left.1 \mathrm{H}\right), 7.98$ $(\mathrm{d}, 1 \mathrm{H}), 7.59(\mathrm{~m}, 3 \mathrm{H}), 7.48(\mathrm{~m}, 2 \mathrm{H}), 7.22(\mathrm{~s}, 1 \mathrm{H}), 7.03(\mathrm{~d}, 1 \mathrm{H}), 5.74(\mathrm{~s}$, $1 \mathrm{H}), 2.96(\mathrm{~s}, 1 \mathrm{H}) .{ }^{13} \mathrm{C}$ NMR $\left(300 \mathrm{MHz}, \mathrm{CDCl}_{3}\right) \delta: 163.95,142.86$, $139.57,132.57,132.15,131.13,129.02,128.63,126.77,124.90$, $124.47,121.59,115.29,114.96,111.90,111.36,29.20$. Anal. calcd for $\mathrm{C}_{17} \mathrm{H}_{13} \mathrm{~F} \mathrm{~N}_{4}$ : C, 69.85; H, 4.48; N, 19.17. Found: C, 69.79; H, 4.48; N, 18.97.

\subsubsection{1-(3-Cyanophenyl)- N-methylimidazo[1,2-a]quinoxalin-4-} amine ( $\mathbf{7 l}$ )

3-Cyanophenylboronic acid (351 $\mathrm{mg}, 2.16 \mathrm{mmol}$ ). Beige solid (84\%). M.p.: $249{ }^{\circ} \mathrm{C} .{ }^{1} \mathrm{H}$ NMR (300 MHz, $\left.\mathrm{CDCl}_{3}\right) \delta: 8.41$ (s, $\left.1 \mathrm{H}\right), 8.13$ (d, $1 \mathrm{H}), 8.00(\mathrm{~d}, 1 \mathrm{H}), 7.77(\mathrm{~d}, 1 \mathrm{H}), 7.64(\mathrm{~m}, 3 \mathrm{H}), 7.51(\mathrm{~m}, 2 \mathrm{H}), 5.77(\mathrm{~s}, 1 \mathrm{H})$, $2.76(\mathrm{~s}, 3 \mathrm{H}) .{ }^{13} \mathrm{C}$ NMR $\left(300 \mathrm{MHz}, \mathrm{CDCl}_{3}\right) \delta: 142.86,139.57,132.25$, 131.64, 131.13, 130.04, 129.02, 128.70, 128.67, 127.96, 127.41, 124.90, 124.47, 119.70, 114.96, 113.80, 29.20. Anal. calcd for $\mathrm{C}_{18} \mathrm{H}_{13} \mathrm{~N}_{5}$ : C, 72.23; H, 4.38; N, 23.40. Found: C, 72.01; H, 4.71; N, 23.25 .

\subsubsection{1-(3-Nitrophenyl)- N-methylimidazo[1,2-a]quinoxalin-4-} amine $(7 \mathrm{~m})$

3-Nitrophenylboronic acid (399 mg, $2.16 \mathrm{mmol}$ ). Yellow solid (95\%). M.p.: $236{ }^{\circ} \mathrm{C} .{ }^{1} \mathrm{H}$ NMR (300 MHz, $\left.\mathrm{CDCl}_{3}\right) \delta: 8.21(\mathrm{~s}, 1 \mathrm{H}), 8.01$ $(\mathrm{d}, 1 \mathrm{H}), 7.70(\mathrm{~m}, 2 \mathrm{H}), 7.58(\mathrm{~d}, 1 \mathrm{H}), 7.40(\mathrm{~m}, 2 \mathrm{H}), 7.17(\mathrm{~m}, 2 \mathrm{H}), 5.17(\mathrm{~s}$, $1 \mathrm{H}), 3.01(\mathrm{~s}, 3 \mathrm{H}) .{ }^{13} \mathrm{C} \mathrm{NMR}\left(300 \mathrm{MHz}, \mathrm{CDCl}_{3}\right) \delta: 147.62,142.86$,
139.57, 131.19, 131.13, 129.02, 127.32, 127.16, 126.99, 126.43, 124.90, $124.66,124.40,122.75,120.76,114.96,29.20$. Anal. calcd for $\mathrm{C}_{17} \mathrm{H}_{13} \mathrm{~N}_{5} \mathrm{O}_{2}$ : C, 63.94; $\mathrm{H}, 4.10 ; \mathrm{N}, 21.93$. Found: $\mathrm{C}, 64.13 ; \mathrm{H}, 3.89 ; \mathrm{N}$, 21.66 .

\subsubsection{1-(3-Furan-N-methylimidazo[1,2-a]quinoxalin-4-amine} (7n)

3-Furanboronic acid (243 mg, $2.16 \mathrm{mmol}$ ). White solid (96). M.p.: $190{ }^{\circ} \mathrm{C} .{ }^{1} \mathrm{H}$ NMR $\left(300 \mathrm{MHz}, \mathrm{CDCl}_{3}\right) \delta: 8.24(\mathrm{~s}, 1 \mathrm{H}), 7.90(\mathrm{~m}, 3 \mathrm{H})$, $7.83(\mathrm{~s}, 1 \mathrm{H}), 7.62(\mathrm{t}, 1 \mathrm{H}), 7.50(\mathrm{~m}, 2 \mathrm{H}), 7.12(\mathrm{~m}, 2 \mathrm{H}), 6.98(\mathrm{~s}, 1 \mathrm{H}), 5.74$ $(\mathrm{s}, 1 \mathrm{H}), 4.85(\mathrm{~d}, 1 \mathrm{H}), 2.85(\mathrm{~s}, 3 \mathrm{H}) .{ }^{13} \mathrm{C}$ NMR $\left(300 \mathrm{MHz}, \mathrm{CDCl}_{3}\right) \delta$ : $141.50,137.73,135.60,130.13,128.79,128.02,124.24,123.70,117.85$, 116.09, 113.61, 107.72, 94.65, 29.20. Anal. calcd for $\mathrm{C}_{15} \mathrm{H}_{12} \mathrm{~N}_{4} \mathrm{O}$ : C, $68.17 ; \mathrm{H}, 4.58$ N, 21.20. Found : C, 68.20 ; H, 4.56 ; N, 21.17.

\subsection{Cell lines and culture techniques}

Melanoma (A375) human cancer cell lines were obtained from American Type Culture Collection (Rockville, Md., USA). Cells were cultured in RPMI medium containing RPMI-1640 (Gibco Laboratories, France), $10 \%$ heat-inactived $\left(56^{\circ} \mathrm{C}\right.$ ) foetal bovine serum (FBS) (Polylabo, Paris, France), $2 \mathrm{mM}$ L-glutamine, $100 \mathrm{IU} / \mathrm{ml}$ penicillin G sodium, $100 \mu \mathrm{g} / \mathrm{ml}$ streptomycin sulphate, and $0.25 \mu \mathrm{g} / \mathrm{ml}$ amphotericin B. Cells were maintained in a humidified atmosphere of $5 \%$ $\mathrm{CO}_{2}$ in air at $37^{\circ} \mathrm{C}$.

\subsection{In vitro cytotoxicity assay}

Previously to the experiments, the number of cells by well, the doubling time and the MTT concentration have been optimized. In all the experiments, A375 cells were seeded at a final concentration of 5000 cells/well in 96-well microtiter plates and allowed to attach overnight. After $20-24 \mathrm{~h}$ incubation, the medium was aspirated carefully from the plates using a sterile Pasteur pipette, and cells were exposed (i) to vehicle controls ( $1 \% \mathrm{DMSO} /$ culture medium and culture medium alone), (ii) fotemustine and imiquimod at concentrations of $10^{-4}-10^{-10} \mu \mathrm{M}$, diluted in the culture medium, and (iii) to the synthesized compounds $\left(10^{-4}-10^{-10} \mu \mathrm{M}\right)$ dissolved in a mixture $1 \% \mathrm{DMSO} /$ culture medium (v/v). After $96 \mathrm{~h}$ of incubation, $10 \mu \mathrm{l}$ of MTT solution in PBS $(5 \mathrm{mg} / \mathrm{ml}$, phosphate-buffer saline $\mathrm{pH}$ 7.3) was added to each well, and the wells were incubated at $37^{\circ} \mathrm{C}$ for $4 \mathrm{~h}$. This colorimetric assay is based on the ability of live and metabolically unimpaired tumor-cell targets to reduce MTT to a blue formazan product. At the end of the incubation period, the supernatant was carefully aspirated, then, $100 \mu \mathrm{l}$ of a mixture of isopropyl alcohol and $1 \mathrm{M}$ hydrochloric acid $(96 / 4, \mathrm{v} / \mathrm{v})$ was added to each well. After 10 min of incubation and vigorous shaking to solubilize formazan crystals, the optical density was measured at $570 \mathrm{~nm}$ in a microculture plate reader (Dynatech MR 5000, France). For each assay, at least three experiments were performed in triplicate.

The individual cell line growth curves confirmed that all A375 lines in control medium remained in the log phase of cell growth $96 \mathrm{~h}$ after plating. Cell survival was expressed as percent of vehicle control. The $\mathrm{IC}_{50}$ values defined as the concentrations of drugs which produced $50 \%$ cell growth inhibition; $50 \%$ reduction of absorbance, were estimated from the sigmoidal dose-response curves.

\section{Acknowledgements}

Special thanks to the Ligue contre le Cancer for its financial support. 


\section{References}

[1] G. Moarbess, C. Deleuze-Masquefa, V. Bonnard, S. Gayraud-Paniagua, J.R. Vidal, F. Bressolle, F. Pinguet, P.A. Bonnet, Biorg. and Med. Chem. 16 (2008)6601-6610.

[2] C. Masquefa, G. Moarbess, P.A. Bonnet, F. Pinguet, A. Bazarbachi, F. Bressolle R.E.P.C.T. WO 2008/063290.

[3] P. Chen, A.M. Doweyko, D. Norris, H.H. Gu, S.H. Spergel, J. Das, R.V. Moquin, J. Lin, J. Wityak, E.J. Iwanowics, K.W. McIntyre, D.J. Shuster, K. Behnia, S. Chong, H. de Fex, S. Pang, S. Pitt, D. Ren Chen, S. Thrall, P. Stanley, O.R. Kocy, M.R. Witmer, S.B. Kanner, G.L. Schieven, J.C. Bzrrish, J. Med. Chem. 47 (2004) 4517-4529.

[4] S.J. Rudy, Dermatol. Nurs. 14 (2002) 268-270.

[5] Y.A. Sidky, E.C. Borden, C.E. Weeks, M.J. Reiter, J.F. Hatcher, G.T. Bryan, Cancer Res. 52 (1992) 3528-3533.

[6] D.N. Sauder, Br. J. Dermatol. 149 (2003) 5-8.

[7] M.J. Reiter, T.L. Testerman, R.L. Miller, C.E. Weeks, M.A. Tomai, J. Leukoc. Biol. 55 (1994) 234-240.

[8] K. Megyeri, W.C. Au, I. Rosztoczy, N.B. Raj, R.L. Miller, M.A. Tomai, P.M. Piha, Mol. Cell. Biol. 15 (1995) 2207-2218.
[9] A. Steinmann, J.O. Funk, G. Schuler, P. Von den Driech, J. Am. Acad. Dermatol. 43 (2000) 555-556.

[10] A.B. Bong, B. Bonnekoh, I. Franke, M.P. Schon, J. Ulrich, H. Gollnick, J. Dermatol. 205 (2002) 135-138

[11] O. Merimsky, M. Inbar, B. Gerard, S. Chaitchik, Melanoma Res. 2 (1992) 401-406.

[12] M. Christmann, M. Pick, H. Lage, D. Schadendorf, B. Kaina, Int J Cancer. 92 (2001) 123-129.

[13] A. Richmond, J. Yang, K. Amitri, P. Dhawan, R.E.P.C.T. WO 2006/0025419.

[14] J.R. Burke, M.A. Pattoli, K.R. Gregor, P.J. Brassil, J.F. MacMaster, K.W. McIntry, X. Yang, V.S. Iotzova, W. Clarke, J. Strnad, Y. Qiu, C. Zusi, J. Biol. Chem. 273 (2003) 1450-1453.

[15] G. Moarbess, H. El-Hajj, K. Kfoury, M.E. El-Sabban, Y. Lepelletier, O. Hermine, C. Deleuze-Masquéfa, P.A. Bonnet, A. Bazarbachi, Blood 111 (2008) 3770-3777. [16] R.E. Tenbrink, R.E.P.C.T. WO 1996/0025414.

[17] S. Parra, F. Laurent, G. Subra, C. Deleuze-Masquefa, V. Benezech, J.R. Frabreguettes, J.P. Vidal, T. Pocock, K. Elliot, R. Small, R. Esacale, A. Michel, J.P. Chapat, P.A. Bonnet, Eur. J. Med. Chem. 36 (2001) 255-264. 